\title{
Sonographically Guided Popliteus Tendon Sheath Injection
}

\section{Techniques and Accuracy}

\author{
Jay Smith, MD, Jonathan T. Finnoff, DO, Borja Santaella-Sante, BS, \\ Troy Henning, DO, Bruce A. Levy, MD, Jim K. Lai, BA
}

\begin{abstract}
Objective. The purpose of this investigation was to describe two sonographically guided popliteus tendon sheath injection techniques and determine their accuracy in a cadaveric model. Methods. A single experienced operator completed 24 sonographically guided popliteus tendon sheath injections, 12 using a longitudinal approach and 12 using a transverse approach relative to the tendon. The injection order was randomized, and all injections were completed with diluted colored latex. Coinvestigators blinded to the injection technique dissected each specimen and graded the colored latex location as accurate (in the sheath), accurate with overflow (within the sheath but also in other regions), or inaccurate (no latex in the sheath). Results. All 12 sonographically guided popliteus sheath injections using the longitudinal approach placed latex into the sheath. Eight of these injections (67\%) also resulted in overflow into the knee joint. Ten of 12 transverse approach injections placed latex into the sheath $(83 \%)$, with 7 of these $(70 \%)$ also producing overflow into the knee joint. Two of 12 transverse injections (17\%) placed latex only into the knee joint and were therefore inaccurate. Conclusions. Sonographic guidance can be used to inject the popliteus tendon sheath with a high degree of accuracy. Although the longitudinal approach is potentially more accurate, both approaches may result in injectate overflow into the knee joint, likely through the popliteus hiatus. Key words: injection; knee; popliteus; sonography.
\end{abstract}

Abbreviations

FCL, fibular collateral ligament; PMTU, popliteus muscletendon unit

Received December 3, 2009, from the Departments of Physical Medicine and Rehabilitation (J.S., J.T.F., J.K.L.), Radiology (J.S.), and Orthopedic Surgery (B.A.L.), Mayo Clinic College of Medicine, Mayo Clinic Sports Medicine Center, Rochester, Minnesota USA; University of Puerto Rico School of Medicine, San Juan, Puerto Rico (B.S.-S.); and Department of Physical Medicine and Rehabilitation, University of Michigan, Ann Arbor, Michigan USA (T.H.). Revision requested December 18, 2009. Revised manuscript accepted for publication December 29, 2009.

This research was funded by the Mayo Clinic Department of Physical Medicine and Rehabilitation.

Address correspondence to Jay Smith, MD, Department of Physical Medicine and Rehabilitation Mayo Clinic College of Medicine, Mayo Clinic Sports Medicine Center, W14, Mayo Building, 200 First St SW, Rochester, MN 55905 USA.

E-mail: smith.jay@mayo.edu

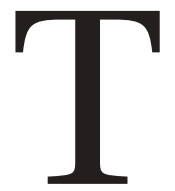

he evaluation and treatment of posterolateral and lateral knee pain remain clinically challenging, particularly in the absence of major trauma. The differential diagnosis is broad and includes iliotibial band syndrome, lateral meniscal conditions (tears, instability, and cysts), osteoarthritis, patellofemoral pain syndrome, lateral plica or fibrous band inflammation, loose bodies, common peroneal (fibular) nerve disorders, biceps femoris tendinopathy, and popliteus tendinopathy. ${ }^{1-4}$ The popliteus muscle-tendon unit (PMTU) arises from the posteromedial tibia and courses toward multiple proximal insertions, the most important of which is its attachment just posterior to the lateral femoral epicondyle, deep and inferior to the fibular (lateral) collateral ligament (FCL). ${ }^{1,5-7}$ Isolated PMTU injuries are uncommon but have been reported as results of minor twisting injuries, tendon instability, impingement against osteophytes or total knee arthroplasty components, calcific tendinopathy, and overuse among runners. ${ }^{1-4,8-18}$ 
Regardless of etiology, isolated conditions affecting the PMTU may be difficult to recognize and treat because of its relatively deep location and close proximity to adjacent structures. ${ }^{4}$ Differential palpation of the popliteus tendon is challenging, and the clinical utility of previously described provocative maneuvers remains indeterminate. ${ }^{2-4}$ The sensitivity and specificity of magnetic resonance imaging and sonography for detecting PMTU injuries have not been formally established, and normal-appearing tendons can still be symptomatic. ${ }^{10,11,19,20}$ Consequently, clinicians have reported the use of popliteus tendon sheath injections for the diagnosis and treatment of patients presenting with PMTU disorders. ${ }^{13,21}$ However, detailed descriptions of these popliteus sheath injection techniques are lacking, and their accuracy has never been formally evaluated. Given the complex regional anatomy and the need for diagnostic and therapeutic precision when performing injections about the lateral knee, we developed two techniques to inject the popliteus tendon sheath using real-time sonographic guidance. Although the sonographic appearance of the normal popliteus tendon has been described, to our knowledge, no previous reports exist that detail and examine the accuracy of sonographically guided popliteus tendon sheath injections..$^{22,23}$

The primary purpose of this investigation was to describe and validate two sonographically guided popliteus tendon sheath injection techniques. We hypothesized that both techniques would place injectate into the popliteus tendon sheath with $100 \%$ accuracy.

\section{Materials and Methods}

\section{General}

The primary author (J.S.) injected the popliteus tendon sheaths of 24 unembalmed cadavers in a randomized fashion using two different sonographically guided techniques: longitudinal and transverse. Injection accuracy was subsequently determined by study coinvestigators blinded to the injection technique. All injections were completed in the Mayo Clinic Procedural Skills Laboratory, and cadaveric specimens were obtained through the Department of Anatomy's Mayo Foundation Bequest Program. Fresh- frozen specimens were fully thawed at room temperature immediately before the study. At the time of the investigation, the senior author had more than 5 years of experience performing musculoskeletal sonography, including sonographically guided popliteus tendon sheath injections. The project was approved by the Mayo Clinic's Bio-Specimens Subcommittee of the Institutional Review Board.

\section{Anatomic Specimens}

Twenty-four unembalmed cadaveric knee specimens were used. No specimen had signs of prior surgery, trauma, or major deformity about the knee. Specimens were labeled 1 through 24, and the age, sex, body mass index, and side (left versus right) was recorded for each donor. Specimens were used for the investigation on the basis of availability and therefore were not necessarily paired (ie, left and right knees from the same donor).

\section{Equipment}

All procedures were completed using an iU22 ultrasound machine with a 17-5 MHz linear array transducer (Philips Healthcare, Bothell, WA), and 25-gauge 38- or 50-mm stainless steel needles.

\section{Injection Procedures}

Each specimen was injected using either a sonographically guided longitudinal approach or a sonographically guided transverse approach, as determined by a computer-generated randomization scheme. All injections consisted of $3.0 \mathrm{~mL}$ of $50 \%$ water-diluted colored latex. Pilot studies determined that this dilution facilitated ease of injection while allowing sufficient latex hardening to assess injectate accuracy via dissection.

The relevant regional anatomy is shown in Figure 1 . The sonographically guided longitudinal popliteus sheath injection was completed as follows: The specimen was placed and stabilized in a lateral decubitus position with the limb flexed to $20^{\circ}$ to $30^{\circ}$ and the leg slightly internally rotated. The lateral femoral epicondyle was palpated, and the cephalad end of the transducer was placed at this point to identify the proximal FCL. ${ }^{22,23}$ After the caudal end of the transducer was rotated to produce a longitudinal view of the FCL, the popliteus sulcus of the lateral femoral condyle was visualized just inferior to the proxi- 
mal FCL attachment. ${ }^{22,23}$ With this transducer orientation, the proximal popliteus tendon appeared within the sulcus in an oblique transverse view (Figure 2). ${ }^{22,23}$ The transducer was then rotated to provide a longitudinal view of the proximal popliteus tendon, including its course around the posterior aspect of the lateral femoral condyle (Figure 3A). Maintaining the longitudinal view of the popliteus tendon, a 25-gauge $50-\mathrm{mm}$ needle was advanced in an oblique cephalocaudal direction parallel to the long axes of both the popliteus tendon and the transducer (Figure 3, B-D). As shown in Figure 3, B and C, the needle typically enters the sheath anterior to the FCL and passes deep to the FCL. In addition, the needle position remains cephalad to the common peroneal (fibular) nerve. Once the needle was advanced into the popliteus sheath, a total of $3 \mathrm{~mL}$ of $50 \%$ water-diluted colored latex was injected while observing sheath distention under direct sonographic guidance.

The sonographically guided transverse popliteus sheath injection was completed as follows: The knee position and initial localization of the femoral sulcus were similar to the longitudinal approach. However, once the proximal popliteus tendon was sonographically visualized within the sulcus, the transducer was rotated to provide a transverse view of the popliteus (Figure 4A). This view was used to provide the opportunity to pass the needle into the deep portion of the popliteus tendon sheath, in the interval between the popliteus tendon and the posterior lateral femoral condyle (achieving this via the longitudinal approach would necessitate needle passage through the tendon). This allows for precise needle placement to treat conditions such as impingement of the popliteus tendon against posterolateral osteophytes or total knee arthroplasty components. ${ }^{1,8,9}$ The needle was advanced from an anterocaudal to a posterocephalad direction, passing through a small amount of skin and subcutaneous tissue before coursing deep to the popliteus tendon (Figure 4, B-D). Similar to the longitudinal approach, the needle typically passed into the sheath anterior to the FCL. Once needle placement was confirmed, a total of $3.0 \mathrm{~mL}$ of $50 \%$ water-diluted colored latex was injected while observing sheath distention under direct sonographic guidance.

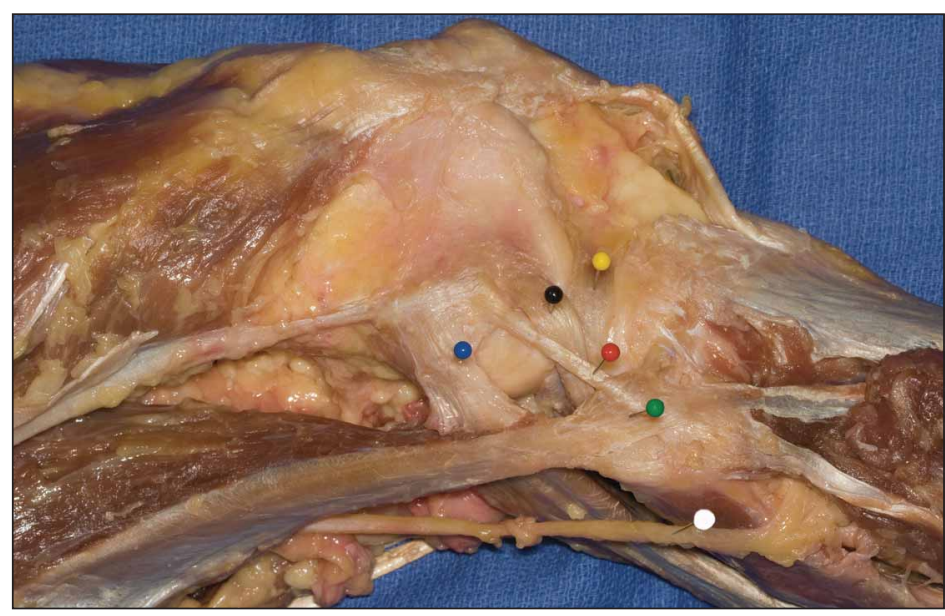

Figure 1. Cadaveric dissection of the lateral knee showing relevant anatomy for sonographically guided popliteus sheath injection. Black indicates popliteus tendon; red, FCL; yellow, lateral meniscus; green, biceps femoris tendon; white, common peroneal (fibular) nerve; and blue, tendon of the lateral head of the gastrocnemius muscle. Note the course of the popliteus tendon deep to the FCL. Left is cephalad; right, caudad; top, anterior; and bottom, deep.

\section{Assessment}

At a minimum of 24 hours after injection, the coauthors dissected each specimen to assess injectate placement. The coauthors were unaware of the technique used for each injectate placement. Injections were graded as accurate (in the popliteus tendon sheath), accurate with overflow (in the popliteus tendon sheath but also elsewhere), or inaccurate (no latex within the popliteus tendon sheath). For those injections that were accurate with overflow or inaccurate, the location of the injectate outside the popliteus tendon sheath was recorded.

Figure 2. Sonographic view of the lateral knee with the transducer placed parallel to the $F C L$. The popliteus tendon $(P)$ is visualized in a transverse view within the femoral sulcus. LM indicates lateral meniscus at the joint line; and TIB, tibia. Left is cephalad; right, caudad; top, superficial/lateral; and bottom, deep/medial.

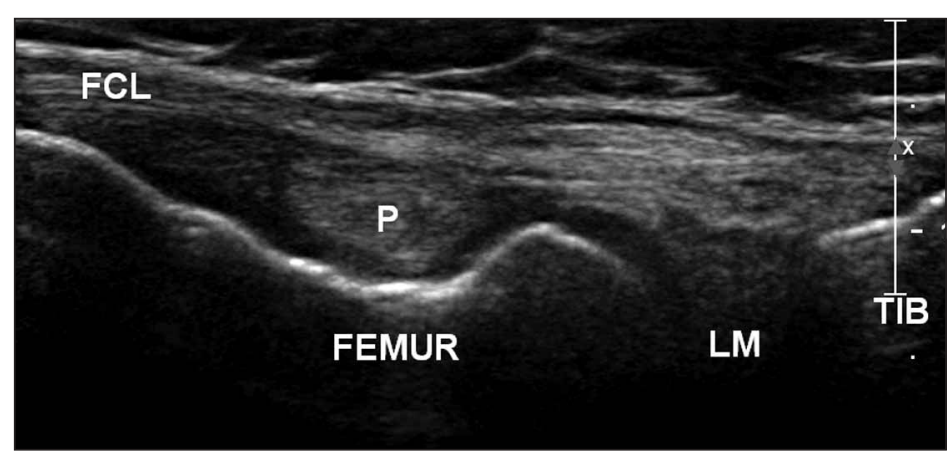




\section{Statistics}

Descriptive statistics were used to report the results of sonographically guided longitudinal and transverse popliteus tendon sheath injections. To determine injection accuracy, the "accurate" and "accurate with overflow" categories were combined and considered "accurate" because of the presence of injectate in the popliteus tendon sheath. For cases in which injections were accurate with overflow or inaccurate, the frequencies with which nontarget structures were affected by the injectate were calculated.

\section{Results}

The 24 cadaveric knee specimens were obtained from 19 separate donors (8 female and 11 male) with a mean age of 79.3 years (range, $56-101$ years) and mean body mass index of $23.4 \mathrm{~kg} / \mathrm{m}^{2}$ (range, $14-39 \mathrm{~kg} / \mathrm{m}^{2}$ ).

Results are shown in Table 1. The longitudinal approach placed latex into the popliteus tendon sheath in $100 \%$ of cases (12 of 12; 95\% confidence interval, $75 \%-100 \%)$. In 8 cases (67\%), some latex was also found in the knee joint. In comparison, the transverse approach placed latex into the sheath in $83 \%$ of cases ( 10 of 12; 95\% confidence interval, $52 \%-98 \%)$. In 7 of these 10 specimens, latex was also found in the knee joint $(70 \%)$. Latex was found only in the knee in 2 specimens (17\%), and these injections were graded as inaccurate. Regardless of the approach (longitudinal or transverse), no injection resulted in latex being found in any location other than the popliteus tendon sheath or knee joint.

Figure 3. A, Longitudinal sonographic view of the popliteus tendon. The transducer position is shown in $\mathbf{B}$. Note the course of the popliteus tendon (POP) around the lateral femoral condyle, outlined by arrows. Left is cephalad; right, caudad; top, superficial/lateral; and bottom, deep/medial. B, Cadaveric dissection showing transducer orientation (box) and needle placement for the longitudinal approach to the popliteus tendon sheath injection. The knee is flexed $20^{\circ}$ to $30^{\circ}$, and the needle passes initially anterior and subsequently deep to the $\mathrm{FCL}$. Red indicates $\mathrm{FCL}$; and black, popliteus tendon. Left is cephalad; right, caudad; top, anterior; and bottom, posterior. C, Magnified view of B showing needle orientation relative to the $\mathrm{FCL}$ (red) and popliteus tendon (black). Note the position of the needle superficial to the popliteus tendon. D, Sonographic view of the popliteus sheath injection using a longitudinal approach. The needle passes adjacent to the superficial popliteus tendon (POP). Arrow indicates tip. The orientation is similar to $\mathbf{A}$.

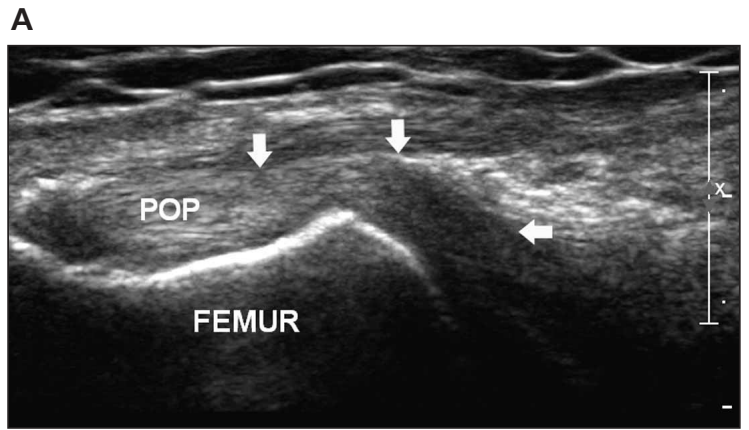

B

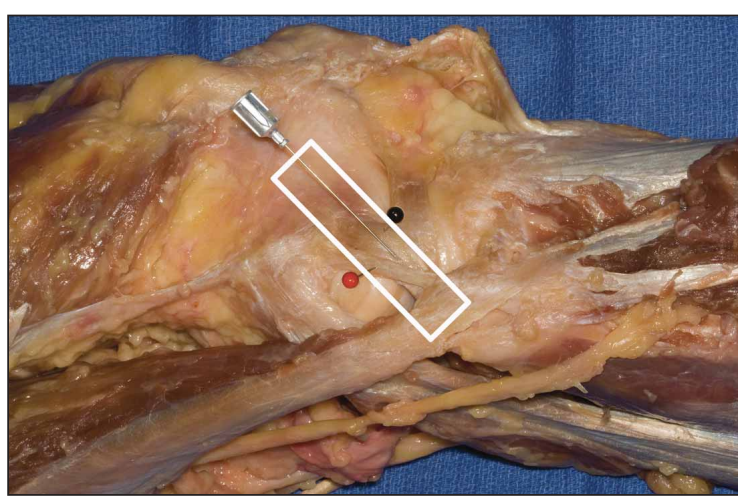

C

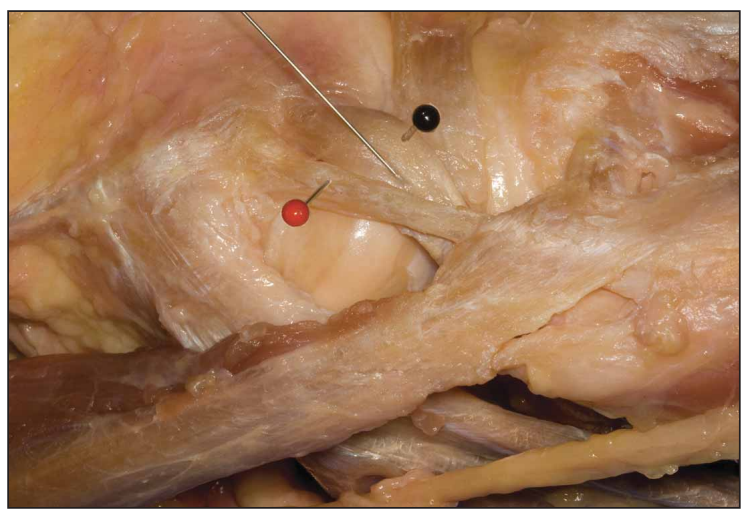

D

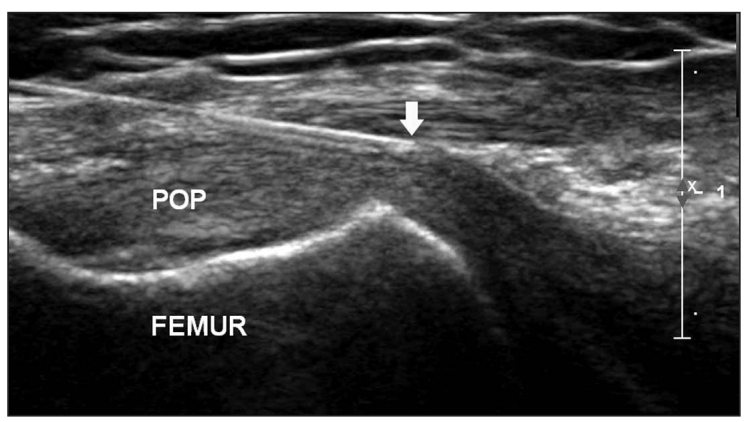




\section{Discussion}

To our knowledge, this investigation represents the first formal description and accuracy assessment of sonographically guided popliteus tendon sheath injection techniques. Our results show that the longitudinal approach can be performed with a high degree of accuracy, placing injectate into the popliteus tendon sheath in $100 \%$ of cases (Table 1). The transverse approach was less accurate, placing injectate into the sheath $83 \%$ of the time, with 2 injections placing latex only within the knee joint. Comparison of our results with previously published data is not possible because to our knowledge, no prior studies exist that assess the accuracy of popliteus tendon sheath injection using palpation or image guidance.

The common finding of latex within the knee joint after sonographically guided popliteus sheath injections warrants further discussion. Sixty-seven percent (8 of 12) of accurate longitudinal and $70 \%$ (7 of 10) of accurate transverse approach injections also placed latex within the knee joint. These findings are not surprising considering that prior studies have shown a normal communication between the popliteus tendon and the knee joint via the popliteus hiatus at the posterolateral tibiofemoral joint. ${ }^{1,3,10,12,24,25}$ Although it is plausible that the size of this normal communication may increase in the postmortem state, the documented presence of this communication in healthy individuals suggests that even accurately placed injections may produce overflow into the tibiofemoral joint.

Figure 4. A, Transverse sonographic view of the popliteus tendon. The transducer position is shown in $\mathbf{B}$. The popliteus tendon (POP) is visualized immediately adjacent to the posterior femoral condyle, its perimeter bordered by arrows. Left is cephalad; right, caudad; top, superficial; and bottom, deep. B, Cadaveric dissection showing transducer orientation (box) and needle placement for the transverse approach to the popliteus tendon sheath injection. The knee is flexed $20^{\circ}$ to $30^{\circ}$, and the needle passes deep to the popliteus tendon (black) into the interface between the tendon and the femur. Red indicates FCL. Left is cephalad; right, caudad; top, anterior; and bottom, posterior, C, Magnified view of $\mathbf{B}$ showing needle orientation relative to the popliteus tendon (black). Note the position of the tip deep to the popliteus tendon. Red indicates FCL. D, Sonographic view of the popliteus sheath injection using a transverse approach. The needle passes deep to the popliteus tendon (POP) adjacent to the femur. The orientation is similar to $\mathbf{A}$.

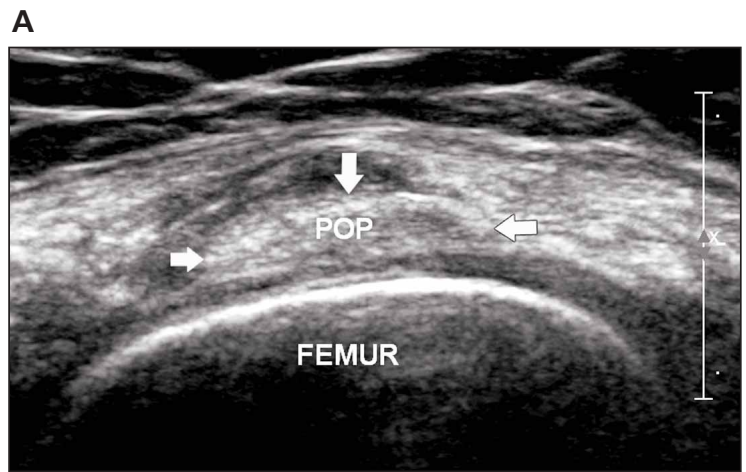

\section{B}

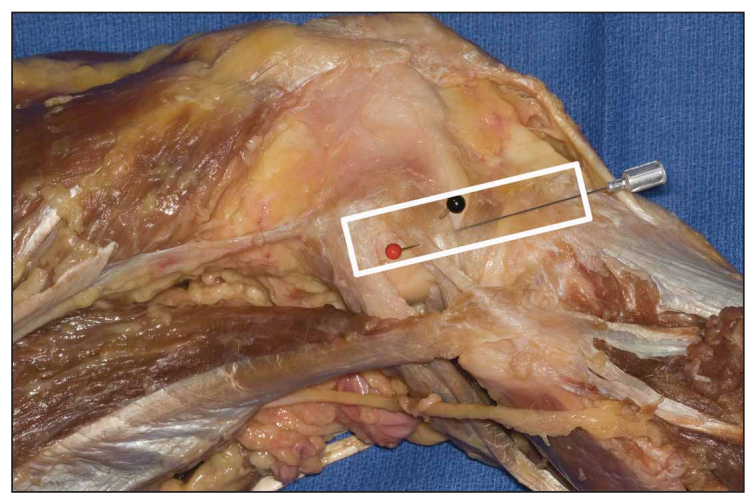

C

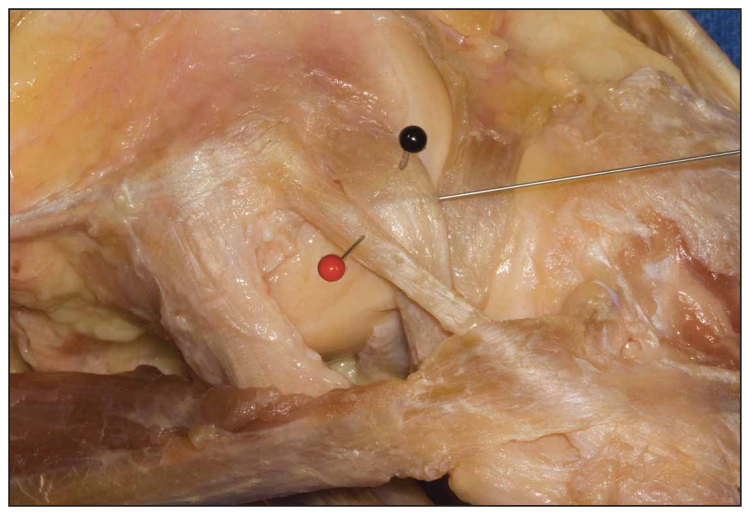

D

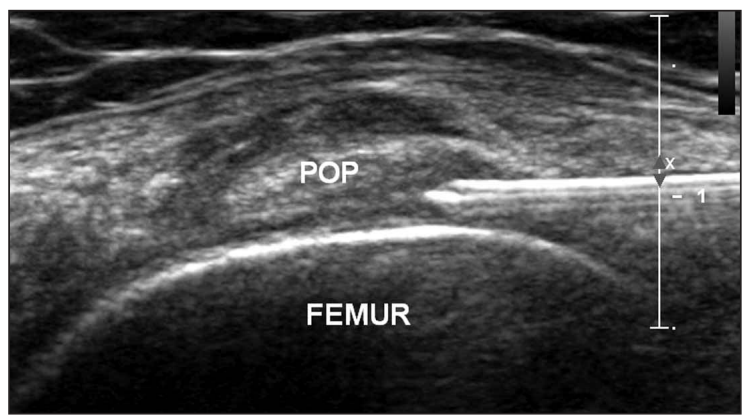


Table 1. Accuracy of Sonographically Guided Popliteus Tendon Sheath Injections

\begin{tabular}{lccc}
\hline Technique & Accurate, $\mathbf{n}(\%)$ & $\begin{array}{c}\text { Accurate } \mathbf{W i t h} \\
\text { Overflow, } \mathbf{n}(\%)\end{array}$ & Inaccurate, $\mathbf{n}(\%)$ \\
\hline Longitudinal approach $(\mathrm{n}=12$ knees) & $4(33)$ & $8(67)$ & $0(0)$ \\
Transverse approach $(\mathrm{n}=12$ knees) & $3(25)$ & $7(58)$ & $2(17)$ \\
\hline
\end{tabular}

This finding would not be unique to a sonographically guided injection in this region compared with accurately placed popliteus tendon sheath injections using palpation guidance or image guidance using other modalities (eg, fluoroscopy). It is possible that the $3-\mathrm{mL}$ volume used for this investigation contributed to the observed overflow. Prior authors have injected volumes ranging from 1.25 to $4 \mathrm{~mL}$ into the popliteus tendon sheath using palpation guidance. ${ }^{13,21}$ In the absence of consensus regarding the optimal injection volume, the $3-\mathrm{mL}$ volume used in this study was determined on the basis of pilot data indicating that $3 \mathrm{~mL}$ reliably produced observable sheath distention using a longitudinal approach. Future investigations may determine whether smaller injectate volumes produce less overflow into the knee joint.

The only 2 inaccurate injections occurred with the transverse approach and placed latex within the knee joint. The primary difference between the longitudinal and transverse approaches is that in the latter case, the needle is placed deep to the popliteus tendon and therefore closer to and potentially within the popliteus

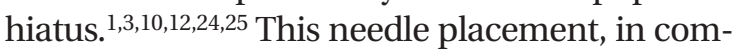
bination with the downward pressure exerted by the popliteus tendon itself, could have contributed to latex flowing into the knee joint via the popliteus hiatus along the path of least resistance. In fact, during multiple transverse approach injections, the senior author visualized low-pressure flow medially and anteriorly toward the popliteus hiatus, despite positioning the limb in slight knee flexion and internal rotation to relax the popliteus. ${ }^{19,26}$

Several clinical implications may be derived from the above discussion. First, regardless of the approach, clinicians must recognize that accurately placed popliteus tendon sheath injections may produce overflow into the knee joint. Clinicians should thus interpret popliteus tendon sheath injection results in the context of the patient's clinical history, physical examination, and available diagnostic imaging (eg, radiography and magnetic resonance imaging) to exclude potential confounding intra-articular pain generators. Second, the specificity of the popliteus tendon sheath injection may be improved by using smaller injection volumes to minimize overflow into the knee joint. Third, the versatility of both the longitudinal and transverse approaches provides the opportunity for more proximal needle placement at the level of the popliteus sulcus. Depositing injectate into this region may reduce overflow posteriorly and medially toward the popliteus hiatus. Although the effects of reduced injectate volume and more proximal needle placement on injectate overflow require further investigation, in clinical practice we typically inject less than $2 \mathrm{~mL}$ total at the level of the proximal popliteus tendon when diagnostic specificity is paramount. If posterolateral impingement is not suspected, a longitudinal approach will typically be used. If femoral condylar osteophyte or total knee arthroplasty component impingement is suspected, a transverse approach will be used, placing injectate at and proximal to the site of impingement. ${ }^{1,8,9}$ This pattern of injectate placement minimizes the possibility of lowresistance overflow into the popliteus hiatus as the path is partially blocked by the region of impingement. Regardless of the approach, injectate flow is sonographically monitored to ensure complete circumferential sheath distention, repositioning the needle as necessary.

Several study limitations are noteworthy. First, clinicians may choose to exercise caution when extrapolating our cadaveric results to patients. Future investigations should explore the clinical utility and technical nuances of applying sonographically guided popliteus tendon sheath injections in diverse clinical populations. Second, this investigation used only 12 unembalmed cadavers for each injection technique. 
Although we think that it is unlikely that injecting more specimens would substantially alter the results of this investigation, we recognize the 95\% confidence intervals for the longitudinal (75\%-100\%) and transverse (52\%-98\%) techniques based on our sample size. Third, although we consider the use of a single experienced operator as a strength of this investigation, it is unknown whether less experienced operators may have similar success with the sonographically guided popliteus tendon sheath injection techniques described herein.

In conclusion, this cadaveric investigation suggests that sonographic guidance can be used to inject the popliteus tendon sheath with a high degree of accuracy. Although the longitudinal approach may be more accurate, both techniques may produce overflow into the knee joint through normal anatomic connections. When indicated, clinicians should consider using sonographic guidance to inject the popliteus tendon sheath for diagnostic or therapeutic purposes.

\section{References}

1. Gaine WJ, Mohammed A. Osteophyte impingement of the popliteus tendon as a cause of lateral knee joint pain. Knee 2002; 9:249-252.

2. Radhakrishna M, Macdonald P, Davidson M, Hodgekinson $\mathrm{R}$, Craton N. Isolated popliteus injury in a professional football player. Clin J Sport Med 2004; 14:365-367.

3. Cooper DE. Snapping popliteus tendon syndrome: a cause of mechanical knee popping in athletes. Am J Sports Med 1999; 27:671-674.

4. Garrick J, Webb D. Sports Injuries: Diagnosis and Management. Philadelphia, PA: WB Saunders Co; 1990.

5. Feipel $\mathrm{V}$, Simonet ML, Rooze M. The proximal attachments of the popliteus muscle: a quantitative study and clinical significance. Surg Radiol Anat 2003; 25:58-63.

6. Pasque C, Noyes FR, Gibbons M, Levy M, Grood E. The role of the popliteofibular ligament and the tendon of the popliteus in providing stability in the human knee. J Bone Joint Surg Br 2003; 85:292-298.

7. Ulrich K, Krudwig WK, Witzel U. Posterolateral aspect and stability of the knee joint, I. Anatomy and function of the popliteus muscle-tendon unit: an anatomical and biomechanical study. Knee Surg Sports Traumatol Arthrosc 2002; 10:86-90.

8. Allardyce TJ, Scuderi GR, Insall JN. Arthroscopic treatment of popliteus tendon dysfunction following total knee arthroplasty. J Arthroplasty 1997; 12:353-355.
9. Barnes $C L$, Scott RD. Popliteus tendon dysfunction following total knee arthroplasty. J Arthroplasty 1995; 10:543545.

10. Mariani PP, Mauro CS, Margheritini F. Arthroscopic diagnosis of the snapping popliteus tendon. Arthroscopy 2005; 21:888-892.

11. Brown TR, Quinn SF, Wensel JP, Kim JH, Demlow T. Diagnosis of popliteus injuries with MR imaging. Skeletal Radiol 1995; 24:511-514.

12. Blake SM, Treble NJ. Popliteus tendon tenosynovitis. Br J Sports Med 2005; 39:e42.

13. Tibrewal SB. Acute calcific tendinitis of the popliteus tendon: an unusual site and clinical syndrome. Ann R Coll Surg Engl 2002; 84:338-341.

14. Mayfield GW. Popliteus tendon tenosynovitis. Am J Sports Med 1977; 5:31-36.

15. Ferrari DA, Wilson DR, Hayes WC. The effect of release of the popliteus insertion and quadriceps force on rotational laxity of the knee. Clin Orthop Relat Res 2003; 412: 225-233.

16. Harner CD, Höher J, Vogrin TM, Carlin GJ, Woo SL. The effects of a popliteus muscle load on in situ forces in the posterior cruciate ligament and on knee kinematics: a human cadaveric study. Am J Sports Med 1998; 26:669673.

17. Davis M, Newsam CJ, Perry J. Electromyographic analysis of the popliteus muscle in level and downhill walking. Clin Orthop Relat Res 1995; 310:211-217.

18. Basmajian JV, Lovejoy JF Jr. Functions of the popliteus muscle in man. A multifactorial electromyographic study. J Bone Joint Surg Am 1971; 53:557-562.

19. LaPrade RF, Gilbert TJ, Bollom TS, Wentorf F, Chaljub G. The magnetic resonance imaging appearance of individual structures of the posterolateral knee: a prospective study of normal knees and knees with surgically verified grade III injuries. Am J Sports Med 2000; 28:191-199.

20. Recondo JA, Salvador E, Villanúa JA, Barrera MC, Gervás C, Alústiza JM. Lateral stabilizing structures of the knee: functional anatomy and injuries assessed with MR imaging. Radiographics 2000; 20(special issue):S91-S102.

21. Petsche TS, Selesnick FH. Popliteus tendinitis: tips for diagnosis and management. Physician Sportsmed 2002; 30:27-31.

22. Barker RP, Lee JC, Healy JC. Normal sonographic anatomy of the posterolateral corner of the knee. AJR Am J Roentgenol 2009; 192:73-79.

23. Sekiya JK, Jacobson JA, Wojtys EM. Sonographic imaging of the posterolateral structures of the knee: findings in human cadavers. Arthroscopy 2002; 18:872-881.

24. Dirim B, Wangwinyuvirat $M$, Frank $A$, et al. Communication between the proximal tibiofibular joint and knee via the subpopliteal recess: MR arthrography with histologic correlation and stratigraphic dissection. AJR Am J Roentgenol 2008; 191:W44-W51. 


\section{Sonographically Guided Popliteus Injection}

25. Fineberg MS, Duquin TR, Axelrod JR. Arthroscopic visualization of the popliteus tendon. Arthroscopy 2008; 24:174-177.

26. LaPrade RF, Ly TV, Wentorf FA, Engebretsen L. The posterolateral attachments of the knee: a qualitative and quantitative morphologic analysis of the fibular collateral ligament, popliteus tendon, popliteofibular ligament, and lateral gastrocnemius tendon. Am J Sports Med 2003; 31:854-860. 